\title{
ON COMPLEMENTED AND ANNIHILATOR ALGEBRAS
}

\author{
by FREDA E. ALEXANDER
}

(Received 15 December, 1967)

1. Introduction. The purpose of this paper is twofold. In [6] Tomiuk gives a representation theorem for a topologically simple right complemented algebra that is also an annihilator algebra. We strengthen this and then give a converse, so as to characterise right complemented algebras among respectively primitive Banach algebras and primitive annihilator Banach algebras. Our second aim is to investigate the relationship between the different annihilator conditions-left annihilator, right annihilator, annihilator, and dual-when imposed on a complemented algebra. Tomiuk [6] has already shown that a right complemented semisimple algebra that is a left annihilator algebra is an annihilator algebra; further, a topologically simple bi-complemented algebra that is also an annihilator algebra is dual. We show that for a topologically simple right complemented algebra all four annihilator conditions are equivalent. Further, for a semi-simple Banach algebra the first three are equivalent provided it is right complemented, and if it is also left complemented, then they are equivalent to duality.

This work was done while the author was a research student at Edinburgh University under the supervision of Professor F. F. Bonsall, whom she would like to thank for his encouragement.

Notation. This is mostly consistent with [4]; however, we follow [1] in denoting by $S_{r}, S_{l}$ the left and right annihilators, respectively, of a subset $S$ of an algebra. The two notations $\mathrm{cl}($ ) and - will denote closure. The following set notations are adopted: (i) if $\mathbf{A}, \mathbf{B}$ are subsets of an algebra, then $\mathbf{A B}=\{a b: a \in \mathbf{A}, b \in \mathbf{B}\}$; (ii) if $\mathbf{S}$ is a subspace of a linear space $\mathbf{V}$ and $\mathbf{T}$ is a set of operators on $\mathbf{V}$, then $\mathbf{T S}=\{t s: t \in \mathbf{T}, s \in \mathbf{S}\}$; (iii) if $a \rightarrow T_{a}$ is a representation of an algebra and $\mathbf{A}$ is a subset of the algebra, then $T_{\mathbf{A}}=\left\{T_{a}: a \in \mathbf{A}\right\}$.

2. Definitions and preliminary lemmas. Let $\mathbf{A}$ be a complex Banach algebra and let $R$ denote the set of all closed right ideals of $\mathbf{A}$. Following [6] we say that $\mathbf{A}$ is a right complemented algebra if there is a mapping $p: \mathbf{R} \rightarrow \mathbf{R}^{p}$ of $R$ onto itself that has the following properties:

$$
\begin{array}{ll}
\mathrm{C}_{1}: \mathbf{R} \cap \mathbf{R}^{p}=(0) & (\mathbf{R} \in R), \\
\mathrm{C}_{2}: \mathbf{R}+\mathbf{R}^{p}=\mathbf{A} & (\mathbf{R} \in R), \\
\mathrm{C}_{3}: \quad\left(\mathbf{R}^{p}\right)^{p}=\mathbf{R} \quad(\mathbf{R} \in R), & \\
\mathrm{C}_{4}: \text { if } \quad \mathbf{R}_{1} \subset \mathbf{R}_{2}, & \text { then } \quad \mathbf{R}_{1}^{p} \supset \mathbf{R}_{2}^{p} \quad\left(\mathbf{R}_{1}, \mathbf{R}_{2} \in R\right) .
\end{array}
$$

The mapping $p$ is called a right complementor on A. Analogously, we define a left complementor and a left complemented algebra. An algebra that is both left complemented and right complemented is said to be bi-complemented.

A Banach algebra is a left annihilator algebra if, for any closed right ideal $\mathbf{R}$ of the algebra, 
$\mathbf{R}_{l}=(0)$ if and only if $\mathbf{R}$ is the whole algebra. A right annihilator algebra is defined analogously. An annihilator algebra is an algebra that is both a left annihilator and a right annihilator algebra. The algebra is dual if, for every closed right (or left) ideal $\mathbf{R}$ (or $\mathbf{L}$ ), $\left(\mathbf{R}_{l}\right)_{r}=\mathbf{R}$ (or $\left.\left(\mathbf{L}_{r}\right)_{l}=\mathbf{L}\right)$.

LеммA 1. In the definition of a right complementor, $\mathrm{C}_{3}$ may be replaced by

$$
\mathrm{C}_{3}^{\prime}:\left(\mathbf{R}^{p}\right)^{p} \supset \mathbf{R} \quad(\mathbf{R} \in R) .
$$

Proof. $\quad \mathbf{R}^{p} \oplus \mathbf{R}=\mathbf{A}=\mathbf{R}^{p} \oplus \mathbf{R}^{p p}$. Hence one inclusion between $\mathbf{R}$ and $\mathbf{R}^{p p}$ will give equality.

Note. It is natural to ask whether $\mathrm{C}_{2}$ can be replaced by $\operatorname{cl}\left(\mathbf{R}+\mathbf{R}^{p}\right)=\mathbf{A}$. This is not possible, as will be demonstrated by the author in a subsequent paper.

Lemma 2. Let $\mathbf{A}$ be a Banach algebra and $p$ a mapping of $R$ into itself that satisfies $\mathrm{C}_{3}^{\prime}, \mathrm{C}_{4}$. If $\left\{\mathbf{R}_{\lambda}: \lambda \in \Lambda\right\}$ is a family of closed right ideals of $\mathbf{A}$, then

$$
\bigcap_{\lambda} \mathbf{R}_{\lambda}^{p}=\left[\operatorname{cl}\left(\sum_{\lambda} \mathbf{R}_{\lambda}\right)\right]^{p} \text {. }
$$

Proof. Write $\mathbf{I}=\bigcap_{\lambda} \mathbf{R}_{\lambda}^{p}$, and $\mathbf{J}=\operatorname{cl}\left(\sum_{\lambda} \mathbf{R}_{\lambda}\right)$. Then, for each $\lambda, \mathbf{R}_{\lambda}^{p} \supset \mathbf{I}$ and so, by $\mathrm{C}_{3}^{\prime}$ and $\mathbf{C}_{4}$, we have $I^{p} \supset \mathbf{R}_{\lambda}^{p p} \supset \mathbf{R}_{\lambda}$. Therefore, since $I^{p}$ is closed, $I^{p} \supset \mathbf{J}$; thus $J^{p} \supset I^{p p} \supset \mathbf{I}$. Conversely, $\mathbf{J} \supset \mathbf{R}_{\lambda}$; therefore, $\mathbf{J}^{p} \subset \mathbf{R}_{\lambda}^{p}$ and hence $\mathbf{J}^{p} \subset \mathbf{I}$. Combining the two inclusions, we have equality. $\operatorname{ain} \mathbf{A}$.

LeMMA 3. Let $\mathbf{A}$ be a right complemented algebra such that $\mathbf{A}_{l}=(0)$. Then $a \in \overline{a \mathbf{A}}$ for all

Proof. Let $a \in \mathbf{A}$ and $\mathbf{R}=\overline{a \mathbf{A}}$. Then $a$ has a unique decomposition $a=a_{1}+a_{2}$, where $a_{1} \in \mathbf{R}$, and $a_{2} \in \mathbf{R}^{p}$. Now, for any $b$ in $\mathbf{A}, a_{2} b=a b-a_{1} b$. However, $a_{2} b \in \mathbf{R}^{p}$ and $a b, a_{1} b \in \mathbf{R}$. Thus $a_{2} b \in \mathbf{R} \cap \mathbf{R}^{p}=(0)$. Therefore, $a_{2} A=(0)$ and, by hypothesis, $a_{2}=0$ and $a \in \mathbf{R}=\overline{a \mathbf{A}}$.

Leмma 4. Every non-zero closed left ideal of a semi-simple right complemented algebra A contains a minimal left ideal.

Proof. From Lemma 2 in [6] it can be seen that, if $\mathbf{M}$ is any maximal modular right ideal of $\mathbf{A}$, there exists a minimal idempotent $\boldsymbol{e}$ that satisfies $\mathbf{M}=(1-e) \mathbf{A}, \mathbf{M}^{p}=e \mathbf{A}$. Now suppose that $\mathbf{L}$ is a left ideal of $\mathbf{A}$ that does not contain a minimal left ideal. Let $\mathbf{M}$ be as above; then $\mathbf{A} e$ is a minimal left ideal. Now, since, for each $u$ in $\mathbf{A}, \mathbf{A} e u$ is either minimal or zero (see the proof of Lemma 2.1.11 in [4]), we have $\mathbf{A} e \mathbf{L}=(0)$. Thus $\mathbf{L} \subset(\mathbf{A} e)_{r}$. However, if $a \in(\mathbf{A} e)_{r}$, then $e a=0$ and so $a=(1-e) a$; therefore $a \in(1-e) \mathbf{A}=\mathbf{M}$. Thus $\mathbf{L} \subset \mathbf{M}$. It follows that $\mathbf{L}$ is contained in every maximal modular right ideal of $\mathbf{A}$ and hence, by the semi-simplicity of $\mathbf{A}$, it is zero.

COROllary. Every minimal-closed left ideal of $\mathbf{A}$ is minimal.

3. Representation theorems for a primitive Banach algebra. In this section $\mathbf{A}$ will denote a primitive Banach algebra with a right complementor $p$. From Lemma 5 in [6] we see that $\mathbf{A}$ 
has a dense socle. Since, in particular, it has minimal one-sided ideals, the discussion in $\S 5$ of Chapter 2 of [4] is relevant. Let $\mathbf{L}=\mathbf{A} e$ be a minimal left ideal and $e$ a fixed minimal idempotent in $\mathbf{A}$. Then the left regular representation $a \rightarrow T_{a}$ of $\mathbf{A}$ on $\mathbf{L}$ is faithful, continuous, and strictly dense. Further, the image of the socle of $\mathbf{A}$ is the set of all elements of finite rank in $T_{\mathbf{A}}$. Using this, together with the density of the socle, we see that $\mathbf{A}$ is topologically simple. The following proposition is implicit in [6].

Proposition 1. For any closed right ideal $\mathbf{R}$ of $\mathbf{A}$ and any closed subspace $\mathbf{S}$ of $\mathbf{L}$,

$$
\mathbf{R} \cap \mathbf{L}=\overline{\mathbf{R L}}=\mathbf{R L} ; \quad \overline{\mathbf{S A}}=\{a \in \mathbf{A}: a \mathbf{L} \subset \mathbf{S}\} \text {. }
$$

Proof. It is clear that $\mathbf{R L} \subset \mathbf{R} \cap \mathbf{L}$ and, since the latter is closed, we have $\overline{\mathbf{R L}} \subset \mathbf{R} \cap \mathbf{L}$. Conversely, if $x \in \mathbf{R} \cap \mathbf{L}$, then $x=x e$ and so $x \in \mathbf{R L}$. The first part of the proposition follows.

Since $\mathbf{S} \subset \mathbf{L}$, we have $\mathbf{S}=\mathbf{S} e$; therefore $\mathbf{S A} \cap \mathbf{L} \supset \mathbf{S} e . \mathbf{A} e=\mathbf{S} . e A e=\mathbf{S} . \mathrm{C} e=\mathbf{S}$. Now, if $z \in \overline{\mathbf{S A}} \cap \mathbf{L}$, then $z=\lim z_{n}$, where $z_{n} \in \mathbf{S A}$. Therefore $z=z e=\lim \left(z_{n} e\right) \in \operatorname{cl}(\mathbf{S A} \cap \mathbf{L}) \subset \mathbf{S}$. Thus $\overline{\mathbf{S A}} \cap \mathbf{L} \subset \mathbf{S}$; since $\mathbf{S}=\mathbf{S} e$, the reverse inclusion is clear and thus we have equality. Therefore $\overline{\mathbf{S A}} \cdot \mathbf{L}=\mathbf{S}$, from which the second part follows.

Now for any closed right ideal $\mathbf{R}$ of $\mathbf{A}$ denote by $S(\mathbf{R})$ the closed subspace $\mathbf{R L}$ of $\mathbf{L}$; for any closed subspace $\mathbf{S}$ of $\mathbf{L}$ denote by $J(\mathbf{S})$ the closed right ideal $\overline{\mathbf{S A}}$ of $\mathbf{A}$. Then, from the corollary to Lemma 10 in [6], it is clear that the map $S$ is one-to-one. Also, from the above proposition, we can see that $S$ maps the set of all closed right ideals of $\mathbf{A}$ onto the set of all closed subspaces of $\mathbf{L}$. It is now easy to verify that $J$ is the inverse map of $S$. Now as in Lemma 6 of [6] it can be seen that the map $p$ defined on the closed subspaces of $L$ by

$$
\mathbf{S}^{p}=S\left([J(\mathbf{S})]^{p}\right)
$$

satisfies the hypotheses of Theorem 1 of [2]. Thus, if $\mathbf{L}$ is infinite dimensional, an inner product may be induced in $\mathbf{L}$ so that (i) for any closed subspace $S$ of $\mathbf{L}, \mathbf{S}^{\perp}=\mathbf{S}^{p}$, or equivalently, for any closed right ideal $\mathbf{R}$ of $\mathbf{A},(\mathbf{R L})^{\perp}=\mathbf{R}^{p} \mathbf{L}$; (ii) $\mathbf{L}$, endowed with the inner product, is a Hilbert space and the resulting norm is equivalent to the given norm. It is clear that the inner product can be varied by any positive real multiplicative constant, and thus we may assume that it is chosen so that $(e, e)=1$.

Notice that, since $\mathbf{A}$ is primitive, the condition that $\mathbf{L}$ be infinite-dimensional is equivalent to the condition that $\mathbf{A}$ itself be infinite-dimensional.

THEOREM 1. Let $\mathbf{A}$ be an infinite-dimensional primitive Banach algebra, and let $p$ be a right complementor on $\mathbf{A}$. Then $\mathbf{A}$ has a faithful, continuous, strictly dense representation $a \rightarrow T_{a}$ on a Hilbert space $\mathbf{H}$. The representation has the following properties:

(i) For any $\mathbf{R} \in R, \mathbf{R}^{p}=\left\{a \in A: T_{a} \mathbf{H} \perp T_{\mathbf{R}} \mathbf{H}\right\}$.

(ii) The socle of $\mathbf{A}$ consists of all elements of $\mathbf{A}$ whose images are of finite rank on $\mathbf{H}$. Its image is generated by the set of all operators of the form $x \otimes y$, where $x$ ranges through $\mathbf{H}$ and $y$ ranges through a subspace $\mathbf{H}_{0}$ of $\mathbf{H}$.

(iii) $\mathbf{H}_{0}$ is dense in $\mathbf{H}$.

(iv) If $E$ is any orthogonal projection on $\mathbf{H}$ and $a \in \mathbf{A}$, then there is an element $b$ in $\mathbf{A}$ satisfying $E T_{a}=T_{b}$ 
Proof. Let $\mathbf{L}=\mathbf{A} e$ be a given minimal left ideal of $\mathbf{A}$ and let $($,$) be the inner product$ induced in $\mathbf{L}$ as in the above discussion. Let $\mathbf{H}$ denote the resultant Hilbert space and let $a \rightarrow T_{a}$ be the representation of $\mathbf{A}$ on $\mathbf{H}$ corresponding to the left regular representation of $\mathbf{A}$ on $\mathbf{L}$. Since $\mathbf{A}$ is semi-simple and $\mathbf{L}$ is minimal, $a \rightarrow T_{a}$ is continuous and faithful and strictly dense. Theorem 2.4.12 in [4] gives (ii). Let $\mathbf{R}$ be any closed right ideal of $\mathbf{A}$. Then, if $a \in \mathbf{R}^{p}$, $a \mathbf{L} \subset \mathbf{R}^{p} \mathbf{L}=(\mathbf{R L})^{\perp}$ and so $T_{a} \mathbf{H} \perp T_{\mathbf{R}} \mathrm{H}$. Conversely, if $T_{a} \mathbf{H} \perp T_{\mathbf{R}} \mathrm{H}$, then $a \mathbf{L} \subset(\mathbf{R L})^{\perp}=\mathbf{R}^{p} \mathbf{L}$; therefore $J([a \mathbf{L}]) \subset J\left(\mathbf{R}^{p} \mathbf{L}\right)=\mathbf{R}^{p}$; thus $a \in \mathbf{R}^{p}$. This completes the proof of (i).

It is clearly sufficient to prove the remainder of the theorem for $\mathbf{A}$ an algebra of operators on a Hilbert space $\mathbf{H}$. A will be the closure of a set of operators of finite rank on $\mathbf{H}$ (since the socle is dense) with respect to a norm \|\| that majorises the operator norm | |. Let $E$ be any orthogonal projection on $\mathbf{H}$, and let $\mathbf{S}$ be its range. Let $\mathbf{R}=\{a \in \mathbf{A}: a \mathbf{H} \subset \mathbf{S}\}$; then $\mathbf{R}^{\mathbf{p}}=\left\{a \in \mathbf{A}: a \mathbf{H} \subset \mathbf{S}^{\perp}\right\}$. Now, for any $a$ in $\mathbf{A}, a=a_{1}+a_{2}$, where $a_{1} \in \mathbf{R}, a_{2} \in \mathbf{R}^{p}$; therefore, for all $h$ in $\mathbf{H}, a h=a_{1} h+a_{2} h$, where $a_{1} h \in \mathrm{S}, a_{2} h \in \mathrm{S}^{\perp}$. But also $a h=E a h+(1-E) a h$, where $E a h \in \mathbf{S},(1-E) a h \in \mathbf{S}$. It follows that $E a h=a_{1} h$. Since this holds for all $h$ in $\mathbf{H}$, we have $E a=a_{1} \in \mathbf{A}$.

We show finally that $\mathbf{H}_{\mathbf{0}}$ is dense in $\mathbf{H}$. If the contrary holds, then there is a non-zero element $x$ of $\mathbf{H}$ that is orthogonal to $\mathbf{H}_{0}$. Therefore, if $a_{n}$ is any element of $\mathbf{A}$ of finite rank, then $a_{n} x=0$. However, if $a \in \mathrm{A}$, there is a sequence $\left\{a_{n}\right\}$ of elements of finite rank such that $\left\|a_{n}-a\right\| \rightarrow 0$; therefore $\left|a_{n}-a\right| \rightarrow 0$, and hence $a x=0$. It follows that $x=0$, and this contradiction establishes (iii).

Note. That the dimension restriction here is necessary can easily be deduced from a counterexample in [2]. By introducing a concept of continuity of a complementor (a property that is found to be automatic in the infinite-dimensional case) the restriction can be relaxed. The author hopes to do this in a later paper.

THEOREM 2. Let $\mathbf{A}$ be as in Theorem 1. Then, if $\mathbf{A}$ is also known to be a left annihilator algebra, the image $T_{\mathbf{A}}$ of $\mathbf{A}$ contains all operators of finite rank on $\mathbf{H}$.

Proof. Let $x$ be any non-zero element of $\mathbf{H}$ and let $\mathbf{S}$ be the set of elements that are orthogonal to $x$. Let $\mathbf{R}=\left\{a \in \mathbf{A}: T_{a} \mathbf{H} \subset \mathbf{S}\right\}$. Then it is clear that $\mathbf{R}$ is a proper closed right ideal of $\mathbf{A}$, and so, by hypothesis, $\mathbf{R}_{l}$ is non-zero. By Lemma 4 , this will contain a minimal left ideal $\mathbf{L}_{0} . \quad \mathbf{L}_{0}$ will be $\left\{a \in A: T_{a}=u \otimes v, u\right.$ varying through $\mathbf{H}, v$ a fixed non-zero element of $\mathbf{H}$ \}. We show that $v$ is a scalar multiple of $x$. Let $h \in \mathrm{S}$; then, since $\mathrm{S}=T_{\mathrm{R}} \mathrm{H}$,

$$
(u \otimes v) h=(h, v) u=0
$$

this holds for all $u$ in $\mathbf{H}$. It follows that $(h, v)=0$ for all $h$ in $\mathbf{S}$, and hence $v \in \mathbf{S}^{\perp}$. Thus $\lambda x \in \mathbf{H}_{0}$ for some non-zero $\lambda$ in $\mathbf{C}$, and, therefore, $x \in \mathbf{H}_{0}$. Thus $\mathbf{H}_{0}=\mathbf{H}$.

Notice that this theorem together with Theorem 1 gives a slightly strengthened form of the representation theorem (Theorem 7) of [6]. We now establish a converse to these two theorems.

THEOREM 3. Let $\mathbf{H}$ be any Hilbert space and $\mathbf{A}$ a strictly dense subalgebra of the algebra $K(\mathbf{H})$ of all compact operators on $\mathbf{H}$; suppose that $\mathbf{A}$ is a Banach algebra under a norm $\|$ \|. Then $\mathbf{A}$ is right complemented if $E \mathbf{A} \subset \mathbf{A}$ for any orthogonal projection $E$ on $\mathbf{H}$. If, further, $\mathbf{A}$ contains all operators of finite rank on $\mathbf{H}$, then $\mathbf{A}$ is an annihilator algebra. 
Proof. A contains minimal one-sided ideals, and so, from Theorem 2.4 .14 in [4], \| \| majorises the operator norm | |. Now for any closed right ideal $\mathbf{R}$ of $\mathbf{A}$ define $\mathbf{R}^{p}$ to be $\left\{a \in \mathbf{A}: a \mathbf{H} \subset(\mathbf{R H})^{\perp}\right\}$. We show first that this is closed. Suppose that $a_{n} \in \mathbf{R}^{p}$ and that $\left\{a_{n}\right\}$ converges to $a$ in $\mathbf{A}$. Then $\left\|a_{n}-a\right\| \rightarrow 0$, and so $\left|a_{n}-a\right| \rightarrow 0$; therefore, since $a_{n} \mathbf{H} \perp \mathbf{R H}$, we have $a \mathbf{H} \perp \mathbf{R H}$ and hence $a \in \mathbf{R}^{p}$. Thus $p$ maps the set of all closed right ideals of $\mathbf{A}$ into itself. Also it is clear that $p$ satisfies $C_{1}$ and $C_{4}$. Again

$$
\mathbf{R}^{p p}=\left\{a \in \mathbf{A}: a \mathbf{H} \subset\left(\mathbf{R}^{p} \mathbf{H}\right)^{\perp}\right\} \supset\{a \in \mathbf{A}: a \mathbf{H} \subset \mathbf{R} \mathbf{H}\} \supset \mathbf{R},
$$

and so $\mathrm{C}_{3}^{\prime}$ holds too.

It is clear that $\left[\operatorname{cl}\left(\mathbf{R}+\mathbf{R}^{p}\right)\right] \mathbf{H}$ contains $\mathbf{R H}$ and $(\mathbf{R H})^{\perp}$ and is therefore the whole of $\mathbf{H}$. Thus, since elements of finite rank are dense in $\mathbf{A}$ and using Theorem 2.4 .18 in [4], we have $\operatorname{cl}\left(\mathbf{R}+\mathbf{R}^{p}\right)=\mathbf{A}$. Thus to establish $\mathbf{C}_{2}$ we need only show that $\mathbf{R}+\mathbf{R}^{p}$ is closed. We notice first that, if $E$ is any orthogonal projection on $\mathbf{H}$, then there exists a scalar constant $k$ such that, for all $a$ in $\mathbf{A},\|E a\| \leqq k\|a\|$. This is a simple consequence of the closed graph theorem and the fact that \|\| majorises | $\mid$. Now let $\left\{a_{n}+b_{n}\right\}$ be a Cauchy sequence in $\mathbf{R}+\mathbf{R}^{p}$ that converges to $c$ in $\mathbf{A}\left(a_{n} \in \mathbf{R}, b_{n} \in \mathbf{R}^{p}\right)$. Let $E$ be the orthogonal projection of $\mathbf{H}$ onto $\mathbf{R H}$. Then $\left\|E a_{n}+E b_{n}-E c\right\| \leqq k\left\|a_{n}+b_{n}-c\right\| \rightarrow 0$. However, for all $h$ in $\mathbf{H}, E a_{n} h=a_{n} h$ and $E b_{n} h=0$; therefore $E a_{n}=a_{n}$ and $E b_{n}=0$. Thus $\left\|a_{n}-E c\right\| \rightarrow 0$. Since $a_{n} \in \mathbf{R}$ for all $n$ and $\mathbf{R}$ is closed, we have $E c \in \mathbf{R}$. Now it follows that $\left\{b_{n}\right\}$ must also be Cauchy and, since $b_{n} \in \mathbf{R}^{p}$, which is closed, $\left\{b_{n}\right\}$ converges to an element $d$ of $\mathbf{R}^{p}$. Thus $c=E c+d \in \mathbf{R}+\mathbf{R}^{p}$. Therefore $\mathbf{R}+\mathbf{R}^{p}$ is closed and $\mathrm{C}_{2}$ follows. Hence, by Lemma $1, p$ is a right complementor.

If $\mathbf{A}$ contains all operators of finite rank on $\mathbf{H}$, then, from Theorem 2.8.23 in [4], $\mathbf{A}$ is an annihilator algebra.

The following corollary is of more practical use in establishing that a given algebra is right complemented.

COROLlaRY. A is also right complemented if the condition on orthogonal projections on $\mathbf{H}$ is replaced by the following: There is a scalar constant $k$ such that $\|E a\|<k\|a\|(a \in \mathbf{A})$ for each orthogonal projection $E$ of finite rank on $H$.

Proof. Define $\mathbf{R}^{p}$ as before. Then it is again clear that \|\| majorises the operator norm and hence that $\mathbf{R}^{p}$ is closed. Also $C_{1}, C_{3}^{\prime}, C_{4}$ hold with unchanged proofs as does the fact that $\operatorname{cl}\left(\mathbf{R}+\mathbf{R}^{p}\right)=\mathbf{A}$.

Now suppose that $\mathbf{R}$ is a closed right ideal of $\mathbf{A}$ that is the topological sum of a family $\left\{\mathbf{R}_{\lambda}: \lambda \in \Lambda\right\}$ of minimal right ideals of $\mathbf{A}$. We show that $\mathbf{R}+\mathbf{R}^{p}$ is closed. For simplicity assume that $k>1$, which can clearly be done. Let $\left\{a_{n}+b_{n}\right\}$ be a Cauchy sequence in $\mathbf{R}+\mathbf{R}^{p}$ and let its limit be $c\left(a_{n} \in \mathbf{R}, b_{n} \in \mathbf{R}^{p}\right)$. Now, given any $\varepsilon>0$, there exists $a_{n, \varepsilon} \in \sum_{\lambda} \mathbf{R}_{\lambda}$ such that $\left\|a_{n, \varepsilon}-a_{n}\right\|<\varepsilon / 2 k$. There exist indices $\lambda(1, n), \ldots, \lambda(r, n) \quad(r=r(n, \varepsilon))$ of $\Lambda$ such that $a_{n, 8} \in \mathbf{R}_{\lambda(1, n)}+\ldots+\mathbf{R}_{\lambda(r, n)}$. Also, since $\left\{a_{n}+b_{n}\right\}$ converges to $c$, there is some $N$ such that $\left\|a_{n}+b_{n}-c\right\|<\varepsilon / k$ for all $n>N$. Let $E_{n, \varepsilon}$ be the orthogonal projection of $\mathbf{H}$ onto

$$
\left(\mathbf{R}_{\lambda(1, n)}+\ldots+\mathbf{R}_{\lambda(r, n)}\right)(\mathbf{H})
$$

then $E_{n, \varepsilon}$ is of finite rank. Also $a_{n, \varepsilon}=E_{n, \varepsilon} a_{n, \varepsilon}$ and consequently 


$$
\left\|a_{n}-E_{n, \varepsilon} a_{n}\right\| \leqq\left\|a_{n}-a_{n, \varepsilon}\right\|+\left\|E_{n, \varepsilon} a_{n}-E_{n, \varepsilon} a_{n, \varepsilon}\right\| \leqq \frac{\varepsilon}{2 k}+\frac{\varepsilon}{2}<\varepsilon
$$

Now let $F$ be the orthogonal projection onto any other finite-dimensional subspace of RH and denote by $F \cup E_{n, \varepsilon}$ the orthogonal projection of $\mathbf{H}$ onto $\left(F \mathbf{H}+E_{n, \varepsilon} \mathbf{H}\right)$; notice that $F \cup E_{n, \varepsilon}$ is also of finite rank. Then

$$
\begin{aligned}
\left\|\left(F \cup E_{n, \varepsilon}\right) a_{n}-a_{n}\right\| & =\left\|E_{n, \varepsilon} a_{n}+\left(F \cup E_{n, \mathrm{e}}\right)\left(1-E_{n, \mathrm{e}}\right) a_{n}-a_{n}\right\| \\
& \leqq\left\|E_{n, \varepsilon} a_{n}-a_{n}\right\|+k\left\|\left(1-E_{n, \mathrm{\varepsilon}}\right) a_{n}\right\| \leqq \varepsilon(1+k) .
\end{aligned}
$$

Also, writing $Q_{n, m}$ for $E_{n, e} \cup E_{m, \varepsilon}$, we have

$$
\left\|Q_{n, m}\left(a_{n}-c\right)\right\|=\left\|Q_{n, m}\left(a_{n}+b_{n}-c\right)\right\| \leqq \varepsilon \text { for } n>N .
$$

Now, taking $F$ to be $E_{m, \varepsilon}$ in (1) and using (2), we have

$$
\begin{aligned}
& \left\|a_{n}-a_{m}\right\| \leqq\left\|Q_{n, m} a_{m}-a_{m}\right\|+\left\|Q_{n, m} a_{n}-a_{n}\right\|+\left\|Q_{n, m}\left(a_{n}-c\right)\right\|+\left\|Q_{n, m}\left(a_{m}-c\right)\right\| \\
& \leqq 2 \varepsilon(1+k)+2 \varepsilon \quad(n, m>N) .
\end{aligned}
$$

Thus $\left\{a_{n}\right\}$ is Cauchy and, as before, converges to an element $a$ of $\mathbf{R}$. Then $\left\{b_{n}\right\}$ is also Cauchy and converges to $b$ in $\mathbf{R}^{p}$. Therefore $c=a+b$ and so is contained in $\mathbf{R}+\mathbf{R}^{p}$. This completes the proof that $\mathbf{R}+\mathbf{R}^{p}$ is closed and therefore establishes $C_{2} . C_{3}$ now follows from Lemma 1 .

We complete the proof by showing that every closed right ideal $\mathbf{R}$ of $\mathbf{A}$ is equal to the topological sum of all the minimal right ideals that it contains. Let $\mathbf{R}$ be any closed right ideal of $\mathbf{A}$ and $\left\{\mathbf{R}_{\lambda}: \lambda \in \Lambda\right\}$ the family of all those minimal right ideals of $\mathbf{A}$ that are contained in $\mathbf{R}$. Let $\mathbf{R}^{\prime}$ be $\operatorname{cl}\left(\sum_{\lambda} \mathbf{R}_{\lambda}\right)$. Then we have shown that $\mathrm{C}_{1}, \mathbf{C}_{2}, \mathbf{C}_{3}$ hold for $\mathbf{R}^{\prime}$. Let $a$ be any element of $\mathbf{R}$; then we may write $a=b+c$, where $b \in \mathbf{R}^{\prime}, c \in \mathbf{R}^{\prime p}$. Also, from Lemma 2 , $\mathbf{R}^{\prime p}=\bigcap_{\lambda} \mathbf{R}_{\lambda}^{p}$. Since $\mathbf{R} \supset \mathbf{R}^{\prime}, b \in \mathbf{R}$ and thus $c \in \mathbf{R} \cap\left(\bigcap_{\lambda} \mathbf{R}_{\lambda}^{p}\right)$. Let $E$ be any one-dimensional projection of $\mathrm{H}$ onto a subspace of $\mathbf{R H}$. Then $E \mathrm{~A}$ consists of elements of rank one whose range is contained in $\mathbf{R H}$. Therefore $E \mathbf{A} \subset \mathbf{R}$ and so $E \mathbf{A}=\mathbf{R}_{\lambda}$ for some $\lambda$ in $\Lambda$. Since $c \in \mathbf{R}_{\lambda}^{p}, c \mathbf{H} \subset\left(\mathbf{R}_{\lambda} \mathbf{H}\right)^{\perp}=(E \mathbf{A H})^{\perp}=(E \mathbf{H})^{\perp}$; thus $c \mathbf{H}$ is orthogonal to every one-dimensional subspace of $\mathbf{R H}$. Therefore, since $c \in \mathbf{R}$, $c h=0$ for all $h$ in $\mathbf{H}$ and so $c=0$. Therefore $a=b \in \mathbf{R}^{\prime}$, and hence $\mathbf{R} \subset \mathbf{R}^{\prime}$. Since the reverse inclusion is clear, we have $\mathbf{R}=\mathbf{R}^{\prime}$. This completes the proof that $\mathbf{A}$ is right complemented.

4. Relationships between annihilator conditions. Let $\mathbf{A}$ be an infinite-dimensional primitive Banach algebra (norm \|\| ) with a right complementor $p$. Let $\mathbf{L}=\mathbf{A} e$ be a given minimal left ideal and let $a \rightarrow T_{a}$ be the representation of $\mathbf{A}$ on $\mathbf{H}$ obtained in Theorem 1 ( $\mathbf{H}$ is a Hilbert space whose elements are those of $\mathbf{L}$ and whose norm | | is equivalent on $\mathbf{L}$ to \|\| ). Then

$$
\mathbf{H}_{0}=\left\{y \in \mathbf{H}: x \otimes y \in T_{\mathbf{A}} \text { for some, and hence all, } x \text { in } \mathbf{H}\right\} .
$$

Now for any $y$ in $\mathbf{H}_{0}$ define

$$
\|y\|^{*}=\|a\|, \quad \text { where } \quad T_{a}=x \otimes y .
$$

Notice that, if $x \in \mathbf{H}$, then $T_{x}=x \otimes e$. 
Lemma 5. $\mathbf{H}_{0}$ is a Banach space with respect to the norm \|\|$^{*}$. On $\mathbf{H}_{0},\|\|^{*}$ majorises both $\|$ \| and | |.

Proof. Consider the minimal right ideal $\mathbf{R}=\left\{a: T_{a}=e \otimes y, y \in \mathbf{H}_{0}\right\}$ of $\mathbf{A}$. The mapping $y \rightarrow a, T_{a}=e \otimes y$ clearly defines an algebraic equivalence between $\mathbf{H}_{0}$ and $\mathbf{R}$. Also, by the definition of \|\|$^{*}$, it is an isometry. However, since $\mathbf{R}$ is minimal, it is closed. Therefore it is clear that $\mathbf{H}_{0}$, with the norm \|\|$^{*}$, is a Banach space.

For any $x$ in $\mathbf{H}_{0},(x \otimes x)(x \otimes x)=(x, x)(x \otimes x)$. Therefore

$$
\begin{aligned}
|x|^{2} \leqq|x \otimes x| & =|(x \otimes e)(e \otimes x)| \quad(\text { since }(e, e)=1) \\
& \leqq|x \otimes e| \cdot|e \otimes x|=|x| \cdot\|x\|^{*} .
\end{aligned}
$$

Hence $|x| \leqq\|x\|^{*}$. Since the norms | |, \|\| are equivalent on $\mathbf{H}$, this also proves that \|\| is majorised by \|\|$^{*}$ on $\mathbf{H}_{0}$.

THEOREM 4. If $\mathbf{A}$ is a primitive Banach algebra with a right complementor $p$, then the following are equivalent:

(i) A is a left annihilator algebra;

(ii) $\mathbf{A}$ is a right annihilator algebra;

(iii) $\mathbf{A}$ is an annihilator algebra;

(iv) A is dual.

Proof. (i) $\Rightarrow$ (ii): If $\mathbf{A}$ is finite-dimensional, then the result is clear, since $\mathbf{A}$ will certainly be an annihilator algebra (any minimal left ideal $\mathbf{L}$ of $\mathbf{A}$ is homeomorphic to the Hilbert space $\mathbf{C}^{n}$, and, by using the argument of the last part of the proof of Theorem 1 , it can be seen that the image of $\mathbf{A}$ under its left regular representation on $\mathbf{L}$ contains all operators of finite rank on $\mathbf{L}$ ). The remaining case follows from Theorems 2 and 3.

(ii) $\Rightarrow$ (iii): Again we have only to consider the infinite-dimensional case. We show first that no proper closed subspace $\mathbf{S}$ of $\mathbf{H}_{0}$ (closure with respect to $\|^{*}$ ) can be dense in $\mathbf{H}$. Let $\mathbf{L}^{\prime}=\left\{a \in \mathbf{A}: T_{a}^{*} \mathbf{H}_{0} \subset \mathbf{S}\right\}$; then $\mathbf{L}^{\prime}$ is a closed left ideal of $\mathbf{A}$. Also $\mathbf{L}^{\prime}$ is proper; for, if $x \in \mathbf{H}_{0}$ but $x \notin \mathbf{S}$, then there exists $a$ in $\mathbf{A}$ such that $T_{a}=x \otimes x$ and hence $a \notin \mathbf{L}^{\prime}$. Therefore, by hypothesis, there is a non-empty closed right ideal $\mathbf{R}$ of $\mathbf{A}$ that annihilates $\mathbf{L}^{\prime}$. Then, for all $h, h^{\prime}$ in $\mathbf{H},\left(T_{\mathbf{L}^{\prime} \mathbf{R}} h, h^{\prime}\right)=(0)$; i.e. $\left(T_{\mathbf{R}} h, T_{\mathbf{L}^{\prime}}{ }^{*} h^{\prime}\right)=(0)$. However, for any $s$ in $\mathbf{S}, h^{\prime} \otimes s \in T_{\mathbf{A}}$ and thus we have $\left(T_{\mathbf{R}} h, \mathbf{S}\right)=(0)$. If $\mathbf{S}$ is dense in $\mathbf{H}$, we deduce from this that $T_{\mathbf{R}} h=(0)$ and, since $h$ is arbitrary in $\mathbf{H}, T_{\mathbf{R}} \mathbf{H}=(0)$, which is the required contradiction.

Now consider the identity mapping $I$ from the normed linear space $\left(\mathbf{H}_{0},\|\|^{*}\right)$ onto the normed linear space $\left(\mathbf{H}_{0},\|\|\right)$. Denote these spaces by $\mathbf{X}_{1}, \mathbf{X}_{2}$ respectively. Then, since $\mathbf{H}_{0}$ is \|\| -dense in $\mathbf{H}$, we have just shown that, if $\mathbf{S}$ is any proper closed subspace of $\mathbf{X}_{1}$, then $I(\mathbf{S})$ is not dense in $\mathbf{X}_{2}$. Now suppose that $\mathbf{T}$ is any maximal closed subspace of $\mathbf{X}_{2}$. Then, since \|\|$^{*}$ majorises \|\|$, I^{-1}(\mathrm{~T})$ is closed. If it is not maximal closed, then it is strictly contained in a proper closed subspace $\mathbf{M}$ of $\mathbf{X}_{1} . I(\mathbf{M}) \supset \mathbf{T}$ and, by the maximality of $\mathbf{T}$, is dense in $\mathbf{X}_{2}$. This contradiction shows that $I^{-1}(\mathbf{T})$ is maximal closed. Conversely, suppose that $\mathbf{S}$ is a maximal closed subspace of $\mathbf{X}_{1} . I(\mathbf{S})$ is not dense in $\mathbf{X}_{2}$ and so it is contained in a proper closed subspace $\mathbf{N}$ of $\mathbf{X}_{2} . I^{-1}(\mathrm{~N})$ is closed and contains $\mathbf{S}$. Therefore, by the maximality of $\mathbf{S}$, it must either be $\mathbf{S}$ or $\mathbf{X}_{1}$. Since the latter is impossible, $\mathbf{N}$ being proper, we have $I^{-1}(\mathbf{N})=\mathbf{S}$ and thus $I(\mathbf{S})$ is closed. By the same argument it can be shown that $I(\mathbf{S})$ cannot be strictly 
contained in any other proper closed subspace of $\mathbf{X}_{\mathbf{2}}$ and is therefore maximal closed. Now, from Lemma $\mathrm{B}$ in [3], $I$ is a homeomorphism. Since $\mathbf{H}_{0}$ is complete with respect to \|\|$^{*}$ and dense in $\mathbf{H}$ with respect to \|\| , we have $\mathbf{H}_{0}=\mathbf{H}$. Thus, by Theorem 2, $\mathbf{A}$ is an annihilator algebra.

(iii) $\Rightarrow$ (iv): By Lemma 3, $a \in \overline{a \mathbf{A}}$ for all $a$ in $\mathbf{A}$. Then, by the proof of Theorem 2.8 .27 in [4], we see that $A$ is dual.

The remaining implications are automatic.

We can extend much of Theorem 4 to the semi-simple case. We note first that, if $\mathbf{A}$ is a semi-simple right complemented algebra, then $\mathbf{A}$ is the direct topological sum of its family of minimal closed two-sided ideals, each of which is a topologically simple right complemented algebra (Theorem 4 in [6]).

THEOREM 5. Let $\mathbf{A}$ be a semi-simple Banach algebra with a right complementor $p$. Then the following are equivalent:

(i) $\mathbf{A}$ is a left annihilator algebra.

(ii) $\mathbf{A}$ is a right annihilator algebra.

(iii) $\mathbf{A}$ is an annihilator algebra.

(iv) Every closed right ideal of $\mathbf{A}$ is an annihilator ideal.

Proof. (i) $\Rightarrow$ (iii): Let $\left\{\mathbf{I}_{\lambda}: \lambda \in \Lambda\right\}$ be the family of all minimal closed two-sided ideals of A. Then each $\mathbf{I}_{\lambda}$ is a topologically simple, semi-simple right complemented algebra, and, from the proof of Theorem 8 in [1], it is also a left annihilator algebra. Then, from Theorem 4, $\mathbf{I}_{\lambda}$ is an annihilator algebra. Now, from Theorem 2.8.29 in [4], $\mathbf{A}$ is an annihilator algebra.

(ii) $\Rightarrow$ (iii): This can be established by a proof analogous to the above with right annihilator replacing left annihilator throughout.

(iii) $\Rightarrow$ (iv): By Theorem 4, each $\mathbf{I}_{\lambda}$ is dual and, by Lemma 3, $a \in \overline{a \mathbf{A}}$ for all $a$ in $\mathbf{A}$. Then, by the proof of Theorem 2.8.29 in [4], it can be seen that every closed right ideal of $\mathbf{A}$ is an annihilator ideal.

This completes the proof, since (iv) $\Rightarrow$ (i) is automatic.

As a corollary we obtain a generalisation of Theorem 9 of [6].

COROLlaRY. If $\mathbf{A}$ satisfies the conditions of the theorem and also some left complementor can be defined on $\mathbf{A}$, then $\mathbf{A}$ is dual.

Proof. Immediate from the analogue for a left complemented algebra of Lemma 3 and Theorem 2.8.29 in [4].

\section{REFERENCES}

1. F. F. Bonsall and A. W. Goldie, Annihilator algebras, Proc. London Math. Soc. (3) 4 (1954), 154-167.

2. S. Kakutani and G. W. Mackey, Ring and lattice characterisations of complex Hilbert space, Bull. Amer. Math. Soc. (2) 52 (1946), 727-733.

3. G. W. Mackey, Isomorphisms of normed linear spaces, Ann. of Math. 43 (1942), 244-260.

4. C. E. Rickart, General theory of Banach algebras (Princeton, 1960).

5. M. F. Smiley, Right annihilator algebras, Proc. Amer. Math. Soc. 6 (1955), 698-701.

6. B. J. Tomiuk, Structure theory of complemented Banach algebras, Canadian J. Math. 14 (1962), 651-659.

\section{UNIVERSITY OF GLASGOW}

